\title{
Optimising Production SySTEMS USING GENETIC ALGORITHMS
}

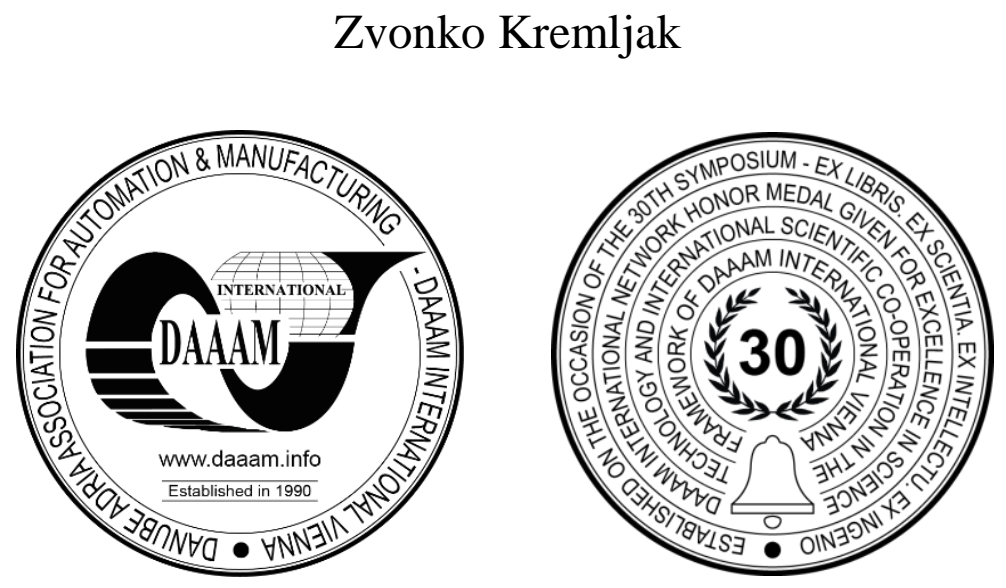

This Publication has to be referred as: Kremljak, Z[vonko] (2019). Optimising Production Systems Using Genetic Algorithms, Proceedings of the 30th DAAAM International Symposium, pp.0139-0147, B. Katalinic (Ed.), Published by DAAAM International, ISBN 978-3-902734-22-8, ISSN 1726-9679, Vienna, Austria DOI: $10.2507 / 30$ th.daaam.proceedings.018

\begin{abstract}
As the most widespread type of evolution algorithms, genetic algorithms (GA) have become a very popular means of heuristically resolving optimisation-related issues. GA have proven extremely powerful particularly in cases when solutions from a wide scope of examination are required. Hitherto, GA have resolved non-deterministic problems. Solutions have not been sought in pre-determined ways and an array thereof has been handled simultaneously which has proven particularly appropriate for problems faced in complex production and business processes. Instead of certainty, order and logical deductions, we are faced with randomisation, evolution and self-organisation.

In this paper, GA are applied on the case-basis of production of aluminium products (variation of production parameters or comparison of equipment layout). The main target of the presented model is to achieve an optimum production plan by taking into consideration dynamic conditions, such as: customer orders, inventories, capacity utilisation rate, quality or raw materials and products and original or cellular layout of equipment.
\end{abstract}

Keywords: Production plan; Genetic algorithms; Modelling; Optimisation; Layout.

\section{Introduction}

In recent years, many manufacturing companies have experienced significant market changes which have and which will affect their business fundamentally and irreversibly. The change from a vendor's to a customer's market. These market changes immediately translate themselves into new production requirements that have a great impact on the production process and the way it is planned and controlled: (i) products change more frequently; (ii) time to market must be reduced; (iii) the number of variants increases to the point where each piece manufactured becomes an individual; (iv) sales and consequently production orders fluctuate significantly; and, last but not least, (v) production costs must be reduced considerably. The pressure on production costs has been further increased because globalisation not only opens new markets to companies but also introduces new competitors. The reduction of production costs itself has a wide range of consequences on the production process. The most important consequence is the requirement for higher productivity over the whole manufacturing period. Understanding the impact that change and disturbances have on the production process is ultimately a quantitative matter. So, the research goal was set to find the optimal production parameters and layout in a selected production facility. Genetic algorithms were used for both problems. 


\subsection{Literature review}

Facility layout problem (FLP) is defined as the placement of facilities in a plant area, with the aim of determining the most effective arrangement in accordance with some criteria or objectives under certain constraints, such as shape, size, orientation, and pick-up/drop-off point of the facilities. Hosseini-Nasab et al. [1] made a review of literature by referring to numerous papers about FLPs. The study was mainly motivated by the current and prospective trends of research on such points as layout evolution, workshop characteristics, problem formulation, and solution methodologies.

Layout problems are common in various manufacturing systems. Actually, the layout problems are related to the location of facilities in a plant, and they have great impact on system performance. A good facility layout can achieve efficient production management, improve production efficiency, and create high economic values. However, real systems are large and complicated, and the computation cost of them is high. Thus, the most of layout problems are NP hard. Zhang and Zhang [2] introduced the notion of survival signature to pre-process the layout problem, which reduces the economic costs and complexity of computation. The optimization of layout solutions is presented by the genetic algorithm (GA). This study demonstrates that the proposed algorithm is effective, and it can be used in solving larger layout problems.

The designer of a plant layout is faced with the efficient arrangement of facilities, the planning of material handling locations, and the aisle design. Klausnitzer and Lasch [3] investigated the advantage of an integrated planning of layout planning subproblems using a holistic view. Several sets of valid inequalities are proposed to shorten the solution time. To compare the solution quality between the proposed integrated approaches and traditional approaches, a comprehensive computational study has been conducted considering several influencing design factors.

In order to solve the problems associated with the organization of the dynamic facility layout in a manufacturing workshop, utilizing a chaotic generic algorithm with improved Tent mapping is proposed by Wei et al. [4] as a solution. The tent map is used to generate the initial population, which is distributed throughout the solution space. Excellent individuals have the Genetic Algorithm optimization with elitist strategy applied to them. Partially matched crossover and mutation operations for single-period-layout encoding string are executed, and adaptive chaotic disturbance is increased to the superior individual. This method is an important innovation in the field of layout optimization by chaotic genetic algorithm. Finally, the paper compares several algorithms by analysing sample outcomes of their respective implementations.

Kalita and Datta presented the single-row facility layout problem, which seeks the arrangement of given facilities along a straight row in such a way that the total material handling cost among the facilities is minimized [5]. It is introduced by instructing to place some facilities in fixed positions, and/or in specified orders with or without allowing the placement of other facilities in between two ordered facilities. Since it would be computationally too expensive for any search technique to satisfy such constraints, a permutation-based genetic algorithm, named as the constrained pGA, is also proposed with some specially designed operators for exploring only feasible solutions.

Stochastic dynamic facility layout problem under demand uncertainty in terms of material flow between facilities was investigated by Peng et al. [6]. A robust approach suggests a robust layout in each period as the most frequent one falling within a pre-specified percentage of the optimal solution for multiple scenarios. Monte Carlo simulation method was used to randomly generate different scenarios. A mathematical model was established to describe the dynamic facility layout problem with the consideration of transport device assignment. As a solution procedure for the proposed model, an improved adaptive genetic algorithm with population initialization strategy was developed to reduce the search space and improve the solving efficiency. Different sized instances were compared with Particle Swarm Optimization (PSO) algorithm to verify the effectiveness of the proposed genetic algorithm.

Part-machine grouping problem is the key step in cell production aiming at grouping parts with similar processing requirements or similar design features into part families and by grouping machines into cells associated to these families. Different techniques for solving it are based on heuristics.

The cell formation problem (CFP) is a crucial component of a cell production design in a manufacturing system. This problem consists of a set of product parts to be manufactured in a group of machines. The objective is to build manufacturing clusters by associating part families with machine cells, with the aim of minimizing the inter-cellular movements of parts by grouping efficacy measures. Pailla et al. presented two approaches to solve the CFP. They presented an evolutionary algorithm that improves the efficiency of the standard genetic algorithm by considering cooperation with a local search around some of the solutions it visits. Additionally, they presented an approach based on simulated annealing that uses the same representation scheme of a feasible solution. To evaluate the performance of both algorithms, they used a known set of CFP instances and compared the results of both algorithms with the results of five other algorithms from the literature [7]. 
Yilmaz and Durmusoglu [8] focused on the batch scheduling problem in multi-hybrid cell manufacturing systems in a dual-resource constrained setting, considering skilled workforce assignment. Cell formation in cellular manufacturing is a critical step to improving productivity by grouping parts and machines. Numerous heuristic search algorithms and several performance measures have been used in finding an effective cell formation solution. This problem consists of finding the sequence of batches on each cell, the starting time of each batch, and assigning employees to the operations of batches in accordance with the desired objective. Three metaheuristics are proposed to solve the batch scheduling problem, namely the genetic algorithm, simulated annealing algorithm, and artificial bee colony algorithm. The main aim of this study was to identify an effective metaheuristic for determining the scheduling and assignment decisions that minimize the average cell response time.

In the study of Sahin and Alpay, a genetic algorithm has been proposed for the problem of part-machine-worker cell formation [9]. The experimental results show that the proposed genetic algorithm can produce optimal solutions for small sized problems and optimal or near-optimal solutions for large sized problems within reasonable times.

To design a cellular manufacturing system, many decisions have to be taken into consideration; grouping machines into cells, determining the system and intracellular layouts, and dividing the resources between the cells. Fahmy proposed in his paper a novel mixed integer linear programming model for the integrated cell formation, cellular layout and worker assignment problem [10]. A genetic algorithm is further proposed to solve the problem, where the chromosome represents locations of machines and assignment of workers to machines. A numerical study is conducted to evaluate the performance of both models.

Shiyas and Pillai presented an algorithm for the design of manufacturing cells and part families [11]. The objective of this algorithm is the maximisation of grouping efficacy, which is one of the most widely used measures of quality for cellular configurations. Assignment of machines to cells is using genetic algorithm, and part assignment heuristic is based on an effective customised rule.

A comprehensive mathematical model is proposed for designing robust machine cells for dynamic part production in the paper written by Deep and Singh [12]. A genetic algorithm based heuristic is proposed to solve the model for minimization of the overall cost considering various manufacturing aspects such as production volume, multiple process route, machine capacity, material handling and subcontracting part operation. A distinctive technique is adopted based on multi-criteria decision technique with significant modifications, which is experimented on test data and compared with published methodology. A novel performance measure is proposed and elaborated analytically to establish its superiority and robustness.

Dmytryshyn et al. presented a new modelling approach called Progressive Modelling and demonstrates it by solving the cell formation problem [13]. In his paper, the Progressive Modelling, a component-based optimization technique, is used to solve the cell formation problem. This novel solution algorithm is utilized to find optimal or near optimal solutions. A benchmark problem in the literature is solved and presented in this paper. The paper concludes by demonstrating the efficiency of the new modelling approach and its future extension.

Karaulova and Shevtshenko developed a work-cells concept supported by a similarity analysis, production line balancing and production process simulation [14]. Simulation allows setting better time distribution for universal work cells.

Zeb et al. presented the hybridization of simulated annealing with genetic algorithm for solving of cell formation problem [15]. The effectiveness of proposed hybrid heuristic algorithm is evaluated through 50 benchmark problems from the literature. The proposed approach achieves the best solutions consistently, within minimum computational time.

The paper of Zupan et al. shows how clusters of products are designed on the basis of a product line data and how an ideal layout optimization is determined on the basis of the intensity of material flow. Layout optimization of a production cell is based on a combination of Schmigalla modified triangular method and the Schwerdfeger circular process [16].

Kong et al. [17] proposed a two-mode modularity clustering method with new similarity measures for parts and machines using an ordinal part-machine matrix. The proposed method considers both incidence and transition among parts and machines and can find an optimal number of clusters. They demonstrated the effectiveness of the proposed method using cell formation problems in comparison with a few existing ones. The result shows that the proposed method produces good cell formation solutions in terms of several performance measures. In addition, they showed a possible application area of the proposed method in process mining, using it to find interpretable clusters of processes and activities from real-life event log data.

One of the main critique on cell production and its algorithms is their inability to handle dynamic events, especially dynamic changes in part spectrum. Agent-oriented computing provides a marvellous opportunity to handle dynamic 
problems and to provide effective solutions. A novel agent-based clustering algorithm for part family formation by considering dynamic demand changes has been proposed by Baykasoglu and Gorkemli [18]. Although the proposed algorithm is not an optimisation-based algorithm and its operation is directed to handle dynamic changes in the problem domain through negotiation, they have shown that it has ability to provide very good results, which are comparable to the best known solutions.

Robotic manufacturing cells entail complex decisions concerning sequencing issues due to uncertainty, which arises in different parameters such as time to failure, time to repair and cycle times that can be effectively supported by computer simulation models. The paper from Vaisi et al. is focused on part sequencing of a two-machine robotic cell in a flow shop, which produces different parts [19]. The study considers machine failures and repair such that cycle time and total production cost should be minimized.

\section{Data set-up and modelling}

Production companies have been increasingly applying contemporary and non-deterministic process and system examination engines. A number of methods are based on natural evolution which can be simulated on the computer and which allow for the examination of a wide variety of solutions (scenarios) within a relatively short timeframe. During simulated evolution, we gradually approach increasingly better solutions (from one generation to the next). This paper applies one of the established evolution methods: genetic algorithms.

The model is composed of the following phases:

- data production and initial set-up of global variables;

- identification of an optimum production plan subject to customer orders;

- scheduling and optimisation of costs;

- inventory optimisation;

- capacity optimisation subject to raw material quality.

The optimisation process is carried out through a tree of logical choices whereby each optimisation task is its own node with various attributes, which help control the process through various rules that enable the achievement of targets within time periods. Due to a lack of time and when the time to make a decision is scarce, various optimisation phases can be carried out as part of routine duty; phase optimisation results can be saved and used as an input. Attribute values can be changed on a global level and can be applied for individual tasks which allows for more ample various simulation options.

The objective of modelling data is to ensure a fast integration path for operational data from the resource planning corporate system and their conversion into the correct format. Data in the genetic algorithm need to be converted into numerical data applied as genes, which compose chromosomes in a sequential order.

The model shall describe the characteristics of the production plant, resource operation, timeframes, rules and conditions. Static data include all data related to the production plant, which do not change frequently, such as resource data (machinery, tools, HR, raw materials) and the underlying production process (including operation routing and description). Static data also include a calendar of shifts and bills of materials. Dynamic data change frequently, are based on customers, suppliers, orders, actual processes in the production plants, inventories, delivery times, etc. Dynamic data are drawn from computer software and are transferred to the production planning system. The link between the two systems is essential and requires a lot of time.

\subsection{Cost function definitions}

The decision whether we are seeking to obtain the maximum, minimum or target value of a specific function is essential and shall be made as soon as the problem examination modelling starts. The model can be built from various aspects, e.g. profits can be defined through a function based on minimum costs or by seeking maximum revenue.

The tree layer architecture of the optimisation engine also allows for the option of precisely setting the cost function and an accurate result. The highest level is the decision tree logic including a task control and attribute value, which can also be applied in decision-making rules. The second level is the genetic optimisation engine with its parameters, chromosomes and genes. The population consists of organisms in the form of points in the space of solutions. The organism consists of genes, which constitute coded values of cost function variable values. Depending on the target function, the GA evaluates the organism generation and applies the following genetic operations: reproduction (selection), hybridisation and mutation. The third level is the program logic required for the optimisation course. 
In our case, it was decided to use the pursuit of maximum profits, based on the production plan, available capacities and customer orders, as the main target function. The main cost function shall identify the optimum production programme, which can be realised depending on fixed customer orders, as restricted by the quality of raw materials (aluminium), available capacities and delivery times. The maximum profit cost function is defined as follows:

$$
\max \left(\sum_{j}\left(c_{j}-b_{j}\right) Q_{j}-C\right)
$$

$c_{j}-$ net sales price of the product or product family,

$b_{j}$ - production costs of the product or product family,

$Q_{j}$ - produced volume of the product or product family,

$C$ - fixed costs.

This way, we simulate an environment, in which a large number of combinations between sales prices $\left(c_{j}\right)$, production costs $\left(b_{j}\right)$, produced volume $\left(Q_{j}\right)$ is examined by the GA and an optimum solution proposed.

In the subsequent optimisation phases, various target functions ensure minimum production costs in various production stages. The planning of capacities is based on the production time for machinery and HR:

$$
\min \left(\sum_{i}\left(t_{m} * C_{m}+t_{w} * C_{w}\right)\right)
$$

$t_{m}$ - processing time at the work centre per work order,

$t_{w}$ - working time at the work centre,

$C_{m}$ - processing time at the specific work centre $i$,

$C_{w}$ - costs of work centre $i$.

Manipulation of inventories and material flow constitutes the following extremely important segment of aluminium production optimised by separate phases whose target is to achieve minimum material costs. The following equation is used for establishing materials management costs:

$$
C_{m}=\sum_{i} \frac{C_{o}+Q_{i} C_{i}}{M_{i}}+\sum_{j} \frac{C_{o}+Q_{j} C_{j}}{M_{j}}
$$

$M_{j}$ - materials costs, including procurement and storage costs,

$C_{o}$ - purchase price,

$Q_{i}, Q_{j}$ - volume of products $i$ and volume of raw materials $j$,

$C_{i}, C_{j}$ - storage costs for products $i$ and raw materials $j$,

$M_{i}, M_{j}$ - total needs for products $i$ and raw materials $j$.

The quality of products and raw materials has a large impact on the moulding and smelting of aluminium alloys. Since the concentration of a specific element in the alloy is contained over an interval, we can identify the most effective composition of elements, specified in the following manner:

$$
\begin{aligned}
& \min \left(\sum_{i} P_{i} \sum_{j}\left(\left(q_{j} \pm d q_{j}\right) * c_{j}\right)\right) \\
& P_{i}-\text { quantity of product } i \\
& q_{j}-\text { normalised consumption of raw materials, } \\
& d q_{j}-\text { tolerance of raw materials } j \text { in alloy } i \\
& c_{j}-\text { raw material costs. }
\end{aligned}
$$

\subsection{Setting up optimisation parameters}

Parameter values shall be applied for testing various strategies of selecting organism pairs on which hybridisation shall be carried out. The size of the population in each evolution cycle shall be specified at the beginning of the optimisation process and initially large enough to facilitate improvement of the cost variable.

According to tests, 50 generations suffice to achieve optimisation results in case studies of similar problems. The termination criterion can be used to terminate the optimisation process as soon as the cost variables become almost static within a specified consecutive number of generations or if the desired level of the cost function has already been achieved. 
The impact of optimisation strategies shall be achieved by the value of parameters specifying the relations between gene hybridisation, reproduction and mutation and the probability of each of these processes. The majority of parameters may be used for increasing the search space and diversity of solutions. A higher probability allows for better GA solutions. Preserving the best organisms speeds up the search process although it needs to be noted that good solutions could be missed.

Problem-based modelling with various types of chromosomes can prove decisive for the quality of the provided solution. The probability to find an optimum solution for a product family is much higher than to find a good solution on a large number of individual products.

\subsection{Restriction definition}

Optimisation, divided into several phases, is subject to various restrictions and limit conditions for each cost function. Essentially, each gene value is restricted by minimum and maximum values, whereas non-sequential chromosomes are restricted by the number of genes in the chromosome.

However, restrictions are rarely defined in the form of a fixed value. The majority thereof is specified by terms defining the interval thereof. In a real situation, conditions are almost always correlated. For example: if the capacity of production is increased, the time of delivery is reduced; if the set-up time and production costs are reduced, the flow of materials is increased, etc.

The calculation of costs in the first optimisation step is subject to maximum profits and the genes are product volumes. After that, solutions are evaluated subject to the impact on the cost function. When we are trying to identify good solutions, it makes sense not to destroy all bad organisms in the generations. Such a limit definition gives us the opportunity to build various search strategies for various purposes, e.g. in some cases, speed is more important than precision. Step-by-step, the entire organism population should draw close to the global optimum.

\section{Research model}

The greater productivity achievement model is based on operational data and shall be verified by various methods. Optimisation phases have been simplified for creating a clearer model, which allows for an easy interpretation of optimisation results. The second reason for that has been the option of resolving the same optimisation problem through various methods.

On some levels, traditional methods have proven extremely efficient and facilitate a simple result interpretation. The main objective of applying various methods for the model (Figure 1) is to verify the model, not to assess the methods applied.

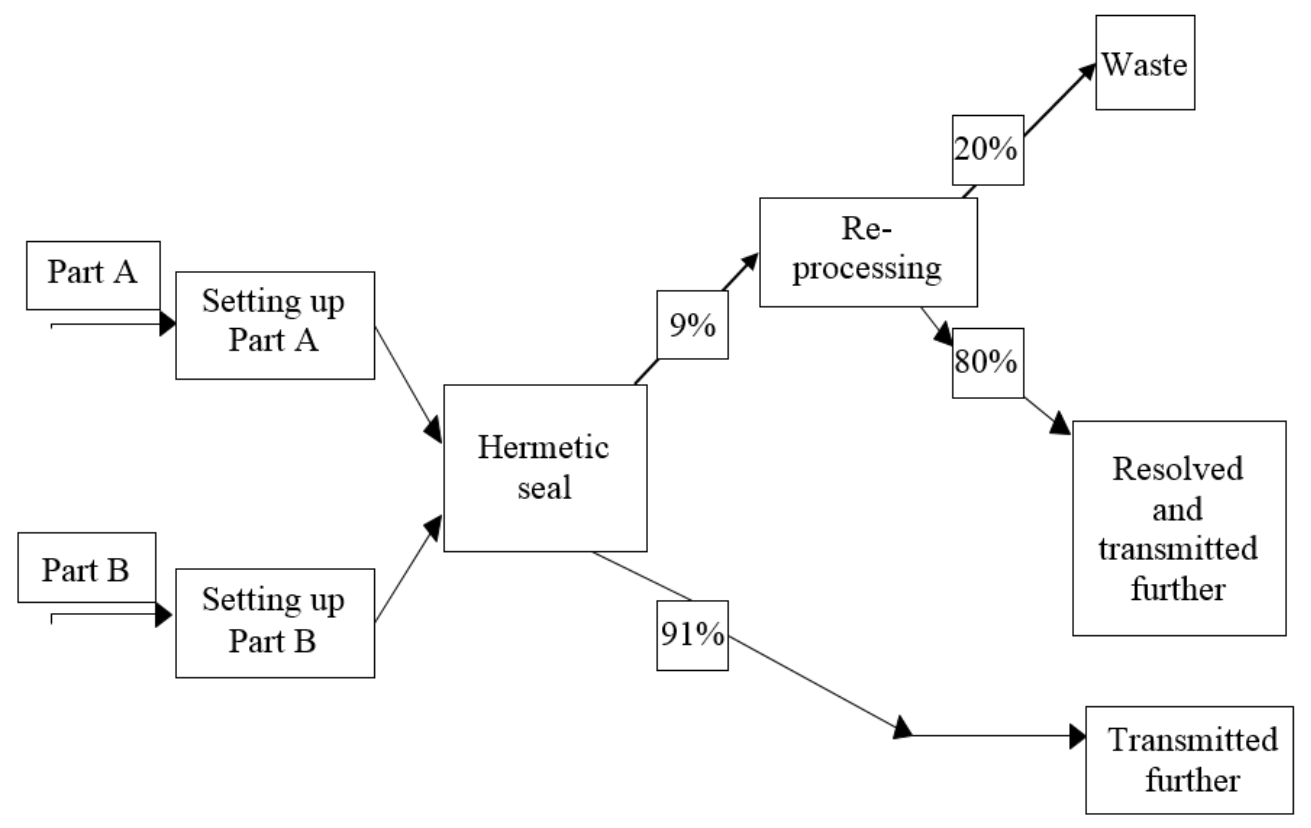

Fig. 1. Model 1 example 


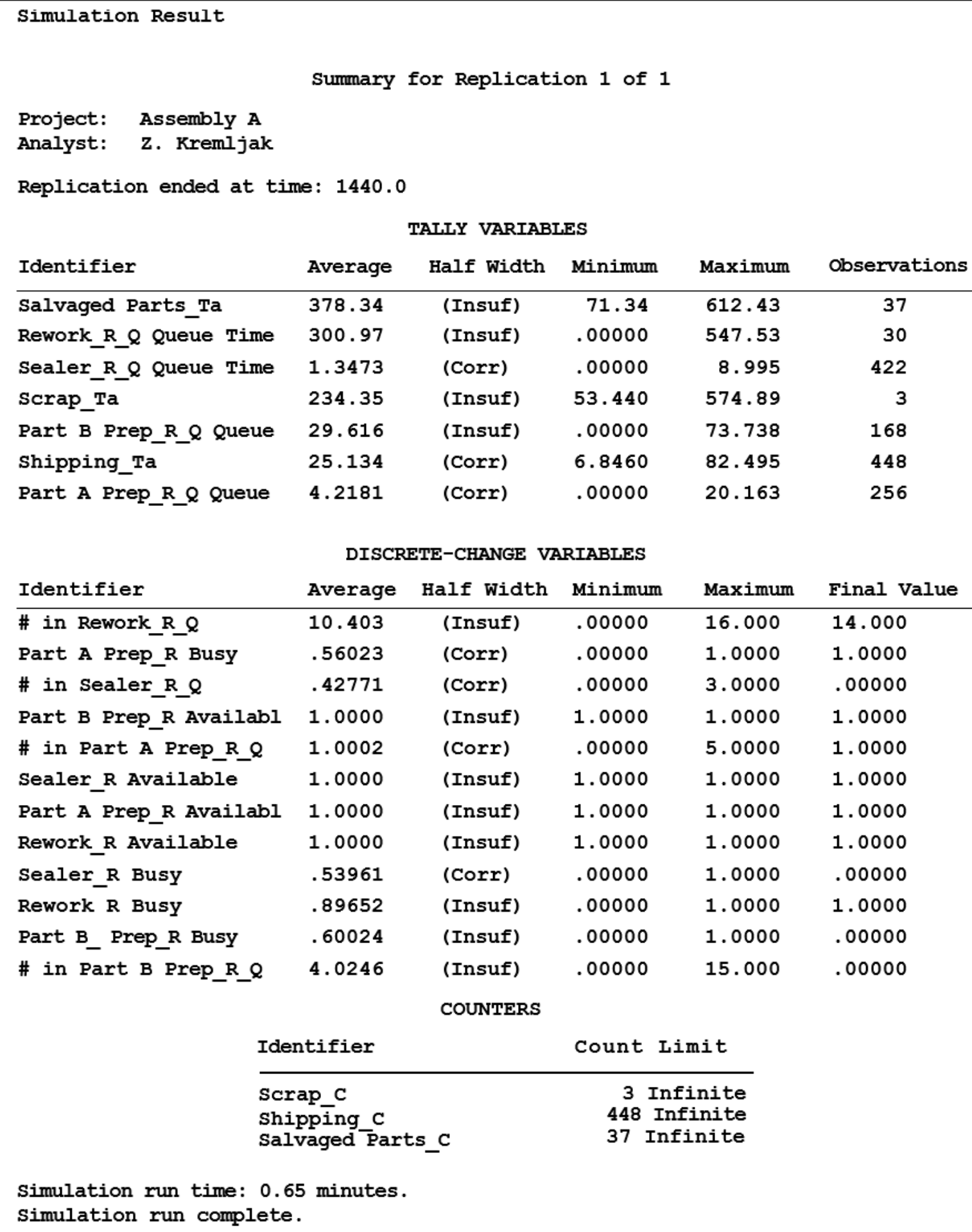

Fig. 2. Example of simulation model results

Generally speaking, the evolution engine of the GA is an extremely time-consuming process whenever it needs to find a problem space for an optimum solution but it has proven extremely efficient in finding almost optimum solutions as early as in the second or third generation. Its true power is demonstrated when the complexity of the model spirals out of control, i.e. when cost functions can no longer be expressed through mathematical equations or if the number of variables is extremely high.

Problem modelling through non-sequential chromosomes lead to a more controlled optimisation when chromosomes stand for various groups of products, types of machines, quality classes and raw material groups. By using this model, some nonsensical results are automatically removed from the search space and the optimisation engine works much faster. 
The cost model used has been applied to evaluate the designed cellular production configuration. This consists of the supposedly most critical cost factors, which are most rapidly available from the producers using cellular production (Table 1). The model takes into consideration investment costs for machinery, machinery start-up and material flow costs. The research model also takes into consideration the impact of other factors which are not included in the model, such as work-in-progress, cycle duration, quality, distribution and productivity of labour, communication and dedication which are much more difficult to evaluate but can also be applied as indicators of the cost reduction or increase trend.

\begin{tabular}{|c|c|c|c|c|c|c|c|}
\hline Recurrence & $\begin{array}{c}\text { Number } \\
\text { of cells }\end{array}$ & $\begin{array}{c}\text { Departments } \\
\text { in the plant }\end{array}$ & $\begin{array}{c}\text { Departments in } \\
\text { the cell }\end{array}$ & $\begin{array}{c}\text { Total set-up } \\
\text { costs }\end{array}$ & $\begin{array}{c}\text { Total } \\
\text { material } \\
\text { costs }\end{array}$ & $\begin{array}{c}\text { Total } \\
\text { investments }\end{array}$ & TOTAL \\
\hline 0 & 0 & 123456 & & 9000 & 1200 & 11000 & 21200 \\
\hline 1 & 1 & 1234 & $(55)$ & 8500 & 1100 & 12000 & 21600 \\
\hline 2 & 3 & & $(12)(34)(56)$ & 7250 & 600 & 12500 & 20350 \\
\hline 3 & 4 & & $(12)(3)(4)(56)$ & 5125 & 600 & 15500 & 21225 \\
\hline 4 & 5 & & $(1)(2)(3)(4)(56)$ & 1500 & 600 & 22000 & 24100 \\
\hline 5 & 6 & & $(1)(2)(3)(4)(5)(6)$ & 0 & 600 & 23000 & 23600 \\
\hline
\end{tabular}

Table 1. Presentation of total costs

Advantages of cellular-organised production are given in Table 2.

\begin{tabular}{|l|c|c|}
\cline { 2 - 3 } \multicolumn{1}{c|}{} & Original layout & Cellular production \\
\hline Productivity per time unit & 50294 products/year & 62967 products/year \\
\hline WIP & 8301 products & 168 products \\
\hline Production cycle & 5 days & 30 minutes \\
\hline
\end{tabular}

Table 2. Results of cellular-organised production

\section{Analysis of optimisation results}

By properly programming production, we can manage a much larger volume of production than before without having to invest in expensive new equipment. The production plant was able to achieve higher productivity levels and higher capacity with existing resources.

Compared to GA, the paper has also applied linear programming (Simplex method) which cannot be fully ignored since it is effective and facilitates a rather simple interpretation of results. The final results of optimisation (expected annual profits) for the discussed case are provided in Table 3.

\begin{tabular}{|l|c|c|c|}
\hline Method & Simplex algorithm & GA 1 sequential & GA 4 non-sequential \\
\hline Profits [EUR] & $11,426,100$ & $10,324,026$ & $12,623,187$ \\
\hline
\end{tabular}

Table 3. Results of searching an optimum production plan through various search methods

A wide range of possible diverse scenarios remains open for further research. Much more than on the calculation method, these affect the modelling method of the optimisation process. Evolution methods have shown great new opportunities for improving the production process in any phase. The optimisation engine provides a new viewpoint to the final user who can simulate various paths or results before making the final decision.

\section{Conclusion}

Evolution algorithms have been present since the early 1960s. So far, genetic algorithms have proven the best and most robust evolution algorithms who seek approximate solutions to challenging problems through principles of inheritance subject to Darwin's paradigm. Genetic algorithms operate on an array of possible solutions, whereby other heuristic algorithms in their iterations apply only one solution. Another important difference also lies in the fact that GA are stochastic and not deterministic algorithms. A proper coding of chromosomes is most likely the most important factor for the performance of GA. They have proven extremely useful in solving complex production problems where traditional methods fail to work and can make key contributions to making strategic business decisions.

More important than an accurately predicted condition, which can be too late, is the way we simulate business conduct as a whole. In particular, we are on the look-out for reasons for poor business decisions on the one hand and opportunities 
which provide us with added value at the same price on the other. By only using one modelling and single-directionoriented thought process, we can quickly miss the actual reason or opportunity. Modelling or looking for various solutions by applying evolution methods can provide us with a completely new view of the existing condition and the reason for poor performance. Future research is going to focus on complementing the system model for applying further evolution methods (Tabu search, PSO, ACO, etc.).

\section{References}

[1] Hosseini-Nasab, H.; Fereidouni, S.; Ghomi, S. M. T. F. \& Fakhrzad, M. B. (2018). Classification of facility layout problems: a review study. International Journal of Advanced Manufacturing Technology, Vol. 94, No. 1-4, pp. 957977, ISSN 0268-3768, DOI:10.1007/s00170-017-0895-8

[2] Zhang, L. \& Zhang, Y.-J. (2019). Solving the facility layout problem with genetic algorithm, $6^{\text {th }}$ IEEE International Conference on Industrial Engineering and Applications, Tokyo, pp. 164-168, ISSN 1662-9795, ISBN 978-1-72810850-6, IEEE

[3] Klausnitzer, A. \& Lasch, R. (2019). Optimal facility layout and material handling network design. Computers \& Operations Research, Vol. 103, pp. 237-251, ISSN 0305-0548, DOI:10.1016/j.cor.2018.11.002

[4] Wei, X.-X.; Yuan, S.-C. \& Ye, Y.-Q. (2019). Optimizing facility layout planning for reconfigurable manufacturing system based on chaos genetic algorithm. Production and Manufacturing Research, Vol. 7, No. 1, pp. 109-124, ISSN 2169-3277, DOI:10.1080/21693277.2019.1602486

[5] Kalita, Z. \& Datta, D. (2018). A constrained single-row facility layout problem. International Journal of Advanced Manufacturing Technology, Vol. 98, No. 5-8, pp. 2173-2184, ISSN 0268-3768, DOI:10.1007/s00170-018-2370-6

[6] Peng, Y.-F.; Zeng, T.; Fan, L.-Z.; Han, Y.-J. \& Xia, B.-X. (2018). An improved genetic algorithm based robust approach for stochastic dynamic facility layout problem. Discrete Dynamics in Nature and Society, Paper ID 1529058, 8 pages, ISSN 1026-0226, DOI:10.1155/2018/1529058

[7] Pailla, A.; Trindade, A. R.; Parada, V. \& Ochi, L. S. (2010). A numerical comparison between simulated annealing and evolutionary approaches to the cell formation problem, Expert Systems with Applications, Vol. 37, No. 7, pp. 5476-5483, ISSN 0957-4174, DOI:10.1016/j.eswa.2010.02.064

[8] Yilmaz, O. F. \& Durmusoglu, M. B. (2018). A performance comparison and evaluation of metaheuristics for a batch scheduling problem in a multi-hybrid cell manufacturing system with skilled workforce assignment. Journal of Industrial and Management Optimization, Vol. 14, No. 3, pp. 1219-1249, ISSN 1547-5816, DOI:10.3934/jimo.2018007

[9] Sahin, Y. B. \& Alpay, S. (2016). A metaheuristic approach for a cubic cell formation problem, Expert Systems with Applications, Vol. 65, pp. 40-51, ISSN 0957-4174, DOI:10.1016/j.eswa.2016.08.034

[10] Fahmy, S. S. (2019). MILP model and a genetic algorithm for the integrated cell formation, layout and worker assignment problem. 10 $10^{\text {th }}$ IEEE International Conference on Mechanical and Intelligent Manufacturing Technologies, Cape Town, pp. 117-121, IEEE

[11] Shiyas, C. R. \& Pillai, V. M. (2014). Cellular manufacturing system design using grouping efficacy-based genetic algorithm, International Journal of Production Research, Vol. 52, No. 12, pp. 3504-3517, ISSN 0020-7543, DOI:10.1080/00207543.2013.871390

[12] Deep, K. \& Singh, P. K. (2015). Design of robust cellular manufacturing system for dynamic part population considering multiple processing routes using genetic algorithm, Journal of Manufacturing Systems, Vol. 35, pp. 155163, ISSN 0278-6125, DOI:10.1016/j.jmsy.2014.09.008

[13] Dmytryshyn, T.; Ismail, M. \& Rashwan, O. (2018). A novel modeling approach for solving the cell formation problem. Proceedings of the ASME International Mechanical Engineering Congress and Exposition, Pittsburgh, Vol. 2, Paper ID V002T02A074, 7 pages, ISBN: 978-0-7918-5200-2, DOI:10.1115/IMECE2018-88128

[14] Karaulova, T. \& Shevtshenko, E. (2015). Work-cells concept development for high mix low volume market conditions, Procedia Engineering, Vol. $100\left(25^{\text {th }}\right.$ DAAAM International Symposium on Intelligent Manufacturing and Automation), pp. 90-99, ISSN 1877-7058, DOI:10.1016/j.proeng.2015.01.346

[15] Zeb, A.; Khan, M.; Khan, N.; Tariq, A.; Ali, L.; Azam, F. \& Jaffery, S. H. I. (2016). Hybridization of simulated annealing with genetic algorithm for cell formation problem, The International Journal of Advanced Manufacturing Technology, Vol. 86, No. 5-8, pp. 2243-2254, ISSN 0268-3768, DOI:10.1007/s00170-015-8288-3

[16] Zupan, H.; Herakovic, N.; Zerovnik, J. \& Berlec, T. (2017). Layout optimization of a production cell, International Journal of Simulation Modelling, Vol. 16, No. 4, pp. 603-616, ISSN 1726-4529, DOI:10.2507/IJSIMM16(4)4.396

[17] Kong, T.; Seong, K.; Song, K. \& Lee, K. (2018). Two-mode modularity clustering of parts and activities for cell formation problems. Computers \& Operations Research, Vol. 100, pp. 77-88, ISSN 0305-0548, DOI:10.1016/j.cor.2018.06.018

[18] Baykasoglu, A. \& Gorkemli, L. (2015). Agent-based dynamic part family formation for cellular manufacturing applications, International Journal of Production Research, Vol. 53, No. 3, pp. 774-792, ISSN 0020-7543, DOI:10.1080/00207543.2014.924634

[19] Vaisi, B.; Farughi H. \& Raissi S. (2018). Two-machine robotic cell sequencing under different uncertainties, International Journal of Simulation Modelling, Vol. 17, No. 2, pp. 284-294, ISSN 1726-4529, DOI:10.2507/IJSIMM17(2)434 